\title{
The Unnecessary Controversy Over Mortality During the First or Second Wave Of COVID-19 Infection in Italy
}

\author{
Marco Bongiovanni* \\ Pneumology Unit, Department of Medicine, Ospedale Salvini, Garbagnate Milanese, ASST Rhodense, Milan, Italy \\ *Corresponding author: Marco Bongiovanni, Pneumology Unit, Department of Medicine, Ospedale Salvini, Garbagnate Milanese, \\ ASST Rhodense, Milan, Italy. \\ To Cite This Article: Marco Bongiovanni. The Unnecessary Controversy Over Mortality During the First or Second Wave Of COVID-19 Infection \\ in Italy. Am J Biomed Sci \& Res. 2021 - 13(2). AJBSR.MS.ID.001855. DOI: 10.34297/AJBSR.2021.13.001855.
}

Received: 眥 May 20, 2021; Published: 㠿 June 17, 2021

\section{Opinion}

COVID-19 pandemic induced dramatic modification of interpersonal relationship and health habit. Both the first and the second wave of the pandemic have been associated with a high morbidity and mortality leading to a serious crisis in the health system in all Countries. Italy, especially the Northern Regions, was one of the first Western Countries to be devastated by this "health hurricane" and most of the subsequent decisions taken in terms of health policy have caused such upheavals in public opinion that they could not even be imaginable before the advent of COVID-19 pandemic. As a consequence, ordinary people have gone from viewing doctors fighting the COVID-19 pandemic as heroes during the first wave to as part of the system preventing freedom of movement by enforcing quarantine measures during the second wave.

The perception of mortality linked to this terrible disease has therefore drastically changed over time. However, not definitive data are at the moment available on the differences in mortality between the two waves of COVID-19 infection. In Italy, morbidity and mortality were very high in both the waves (the first one between March and May 2020, the second one between September and December 2020). Different reasons at the basis of this specific finding have been hypothesized [1,2]; in particular, during the first wave, the advanced age of patients hospitalized for COVID-19 infection in Italy and their multiple co-morbidities were considered as possible explanations, as was the limited number of intensive care unit beds in the Northern Regions of Italy that proved to be insufficient to provide to all the patients who needed intensive therapies. Further, during the first wave of pandemic the number of subjects tested for SARS-CoV2 infection was very limited, as the indication provided by the health authorities was not to test individuals with mild to moderate symptoms; in fact, such individuals were only put in quarantine for 14 days without being tested and this indication may have played a role in further propagating the pandemic. After the first wave of pandemic, several different health measures have been taken to prevent the second wave of pandemic: in some hospitals the total number of ICU beds were augmented and also the primary care doctors were much more involved in the initial management and diagnosis of patients with possible COVID-19 infection; moreover, a greater number of nasopharyngeal swabs was available in order to test all the subjects with clinical symptoms related to COVID-19 infection [3]. Recently, some papers evaluated the differences in morbidity and mortality of COVID-19 infection in Italy, trying to find more or less convincing explanations for the results obtained.

In a paper recently published by Borghesi et al. [4], the authors stated that the second wave of COVID-19 infection in Italy seems to be "less severe and deadly" than the first one. This conclusion was based on a comparison made on 100 consecutive patients admitted to their hospital during the first and the second wave and was measured in terms of severity of COVID-19 pneumonia by using chest X-ray and other clinical parameters such as patient's outcome, co-morbidities, corticosteroid use and C-reactive protein (CRP) levels. They found that chest X-ray severity, CRP levels and in-hospital mortality were all statistically higher in the first than in the second wave.

Another study published by our group, though with different methods and parameters used, found similar results [5]. In this paper, we compared data on patients admitted to the emergency 
room and diagnosed with COVID-19 infection during two different timeframes: the first one between 2 and 8 March 2020 (first wave) and the second between 19 and 25 October 2020 (second wave). These periods were chosen because they represented the week before the establishment of the red zone in Italy. Overall, 60 patients with COVID-19 infection were evaluated in the emergency room during the first wave and 232 during the second wave, without significant differences according to gender, age and co-morbidities at initial presentation. Nevertheless, patients admitted during the first wave had more severe symptoms leading to an increased risk of oro-tracheal intubation, not-invasive ventilation and, finally, to an increased risk of death.

Though a higher percentage of subjects was admitted to the ward during the first wave, the overall number of patients hospitalized was higher during the second wave (140 vs 57 patients). Taken together, these data demonstrated that though the severity and the mortality of COVID-19 infection was lower during the second wave, an overall larger number of patients was hospitalized, albeit with milder symptoms. A possible explanation for these findings might be due to the fear and worry of the population for what happened during the first wave, so that people went to the emergency room at an earlier stage of infection and with milder symptoms. As a consequence, the global impact on the health management was significantly higher during the second wave of COVID-19 pandemic, with a larger number of patients being hospitalized.

However, a simple comparison of numbers concerning COVID-19 related deaths in Italy during the two waves seems to show the contrary. According to the COVID-19 Special report provided by the Graduate School of Health Economics and Management (ALTEMS) and to the "Worldometer country" database, the number of deaths in Italy was higher during the second wave $(n=38,549$ for ALTEMS, $\mathrm{n}=38535$ for Worldometer) than that occurred during the first wave ( $n=34,167$ for ALTEMS and $n=34260$ for Worldometer) [6,7]. Other indicators of severity of disease except deaths, such as the incidence of new daily infection cases, the infection fatality rate (IFR), and the case fatality ratio (CFR), which is the number of deaths divided by the number of confirmed cases, have been used; however, these indicators are mainly based on the number of infected cases, that sometimes is unknown as many infection cases remained untested, as occurred especially during the first wave of pandemic making questionable the correct estimation of IFR and CFR [8,9]. For these reasons, some authors suggested that the overall number of deaths could be considered a good proxy of new daily COVID-19 infection cases and a reliable indicator of the burden on the hospitals with the risk of a consequent sub-optimal care [10]. Further, in literature there is a linear relationship between the number of infection cases and the number of COVID-19 related deaths that also correlates with the severity of cases admitted in hospital $[11,12]$.
Taken together, all these observations on the mortality of COVID-19 infection are quite controversial and difficult to explain. But the main question arising from these data is: it really is so important to establish if the mortality was higher during the first or the second wave? Are more than 38000 deaths so different from more than 34000 ? What we doctor who have dealt with and still deal with COVID-19 infection should recognize is that the advent of this disease has drastically changed the way we work and has induced global changes in the management of patients and in the healthcare systems that are likely to be maintained also in the future. Moreover, the impact that COVID-19 infection has had on the whole World represents such an epochal event that is will certainly influence also the future of humanity.

\section{Acknowledgement}

None.

\section{Conflicts of interest}

No conflicts of interest.

\section{References}

1. Onder G, Rezza G, Brusaferro S (2020) Case-fatality rate and characteristics of patients dying in relation to COVID-19 in Italy. JAMA 323(18): 1775-1776.

2. Immovilli P, Morelli N, Antonucci E, Guido Radaelli, Mario Barbera, et al. (2020) COVID-19 mortality and ICU admission: the Italian experience. Crit Care 24(1): 228.

3. Bongiovanni M (2021) Disputes in the management of COVID-19 infected subjects. Health and Primary Care (5): 1-2.

4. Borghesi A, Golemi S, Carapella N, Angelo Zigliani, Davide Farina, et al. (2021) Lombardy, Northern Italy: COVID-19 second wave less severe and deadly than the first? A preliminary investigation. Infect Dis 53(5): 370-375.

5. Bongiovanni M, Arienti R, Bini F, et al. (2021) Differences between the waves in Northern Italy: how the characteristics and the outcome of COVID-19 infected patients admitted to the emergency room have changed. J Infect S0163-4453(21): 00210-3.

6. (2020) ALTEMS. Instant Reports.

7. Worldometer. Coronavirus Update (Live): 123,555,602 Cases and $2,723,780$ Deaths from COVID-19 Virus Pandemic-Worldometer (worldometers.info).

8. Meyerowitz Katz G, Merone L (2020) A systematic review and metaanalysis of published research data on COVID-19 infection fatality rates. Int J Infect Dis 101: 138-148.

9. Chirico F, Nucera G, Magnavita N (2020) Estimating case fatality ratio during COVID-19 epidemics: Pitfalls and alternatives. J Infect Dev Ctries 14(5): 438-439.

10. Khalili M, Karamouzian M, Nasiri N, Sara Javadi, Ali Mirzazadeh, et al. (2020) Epidemiological characteristics of COVID-19: a systematic review and meta-analysis. Epidemiol Infect 148: e130.

11. Patel U, Malik P, Mehta D, Dhaivat Shah, Raveena Kelkar, et al. (2020) Early epidemiological indicators, outcomes, and interventions of COVID-19 pandemic: A systematic review. J Glob Health 10(2): 020506.

12. Mannucci E, Silverii A, Monami M (2021) Association between different screening strategies for SARS-CoV-2 and deaths and severe disease in Italy. Int J Clin Pract 75(4): e13867. 